\title{
Modelling the impact of migration on HIV persistency in Ghana
}

\author{
Ofosuhene O. Apenteng ${ }^{1, *}$, Noor Azina Ismail $^{2}$ \\ ${ }^{1}$ Research Group for Genomic Epidemiology, National Food Institute, Technical University of Denmark, Denmark. \\ ${ }^{2}$ Department of Applied Statistics, Faculty of Economics \& Administration, University of Malaya, Kuala Lumpur, Malaysia.
}

\begin{abstract}
Migrants may be exposed to health risks before, during and after leaving their countries of origin. Unfortunately, knowledge about the health status of migrants is often limited because they are often excluded from surveys. This paper extends the susceptible-exposed-infective-removed model to handle the assumption of homogeneous mixing, the incorporation of migration and the induced death rates of the disease in modelling the spread of HIV/AIDS. These extensions demonstrate that the impact of migration on HIV persistency is critical when attempting to predict where and how fast the disease will propagate. The spectral analysis of a time series was used to determine the frequency at which the disease is spread and its equilibrium levels. The results indicate that with the persistent flow of migration into a country, the disease status changes from epidemic to endemic. If the direct flow of migration into the population is restricted, the persistent spread of the disease can be minimised.
\end{abstract}

Keywords HIV/AIDS, $S I_{1} I_{2} A$ Model, Migration, Mathematical Transmission Modelling, Simulation

DOI: $10.19139 /$ soic.v7i1.552

\section{Introduction}

The human immunodeficiency virus (HIV) infection, which leads to acquired immunodeficiency syndrome (AIDS), has become a problematic infectious disease in both the developed and developing countries. AIDS is one of the most serious and deadly diseases in human history and is caused by the human immunodeficiency virus (HIV) [1]. In many African countries, AIDS is already a major cause of death [2,3], and it is predicted by experts that it will soon become so in Asian countries having larger scale populations [4]. It is well known that the HIV virus has a long incubation and infectious period. It is now known that migration plays a vital role in disease studies $[5,6,7,9]$. There is a growing need to model the effects of environmental factors, including migration patterns, on the spread of AIDS to gain an increased understanding of how AIDS spreads [11, 8]. Therefore, possible ways to combat that spread, not just through drugs but also through enhanced environmental control, can be proposed. Such models can lead to novel hypotheses and predictions that can be grounded in the data available to health organisations responsible for modelling or controlling the spread of AIDS. For example, recent events in Ghana indicate that there is an increase in immigration issues in the country that could pose a high risk of spread of disease [12]. According to the Ghana Immigration Service (GIS) more people migrated into Ghana than from Ghana in the period from 2000 to 2006 [13]. In 2012, the major inflow of asylum of refugees alone was 9,140 thousand people in Ghana [14]. For the past decades, the migration of health experts from Ghana has been a cause for concern. This concern has become so important that the effect of migration flow on the spread of HIV modelling is being considered. The motivation of this paper is to investigate how migration has affected the spread of HIV. This is because it has become one of the attributes that contribute to the feared and devastating diseases which considerably

*Correspondence to: Ofosuhene O. Apenteng (Email:ofap@food.dtu.dk). Research Group for Genomic Epidemiology, National Food Institute, Technical University of Denmark, Kemitorvet, 2800, Kgs. Lyngby, Denmark.

ISSN 2310-5070 (online) ISSN 2311-004X (print)

Copyright (C) 2019 International Academic Press 
affected the human population. It is important to mention that to our best of knowledge this research work is different from some the previous work. The novelty of our study is that we shall deal with mathematical models have ever been done before in Ghana on migration and HIV with mathematical modelling. That notwithstanding, this study assessed the effect of migration on the spread of HIV in Ghana. In addition, the objective of this paper is to demonstrate how the impact of the external net migration of people on the persistent spread of HIV in Ghana. The empirical results of the persistent external migration are compared to the results without the flow of external migration from the country. Spectral analysis is used to determine the rate at which the HIV spreads with the flow of external migration.

\section{Mathematical Model}

We used compartmental models to understand the effects of persistent external migration of the spread and the control of HIV in Ghana. We initially made an assumption of homogeneous mixing among the entire high risk population for simplicity. We then formulated a nonlinear mathematical model to describe the impact of persistent immigration on the spread of HIV. We used these formulated models to comprehensively understand the HIV epidemic in Ghana and to explore policy related questions, including an investigation of the impact of migration on HIV persistency and HIV prevention interventions in different provinces in Ghana. It is assumed that susceptible become infected via sexual contacts and that all of the infectives eventually developed AIDS.

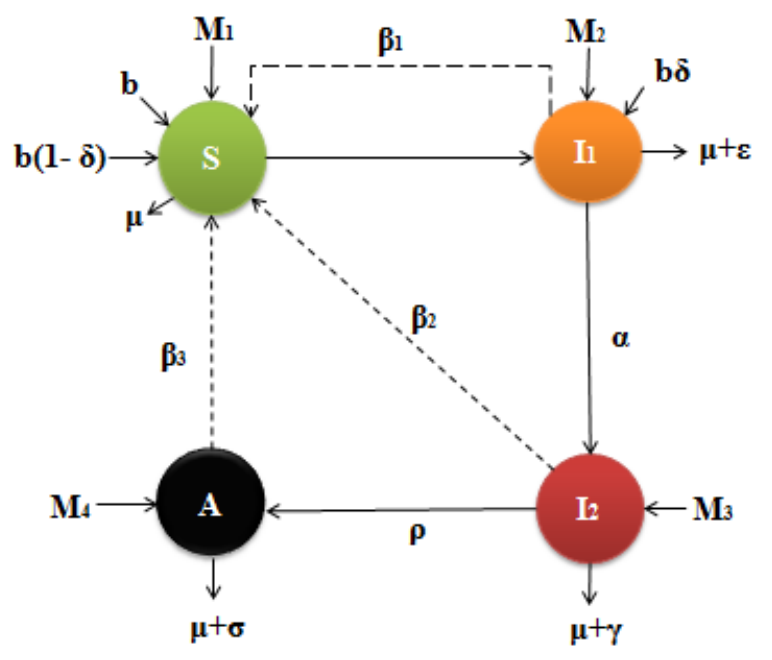

Figure 1. $S I_{1} I_{2} A$ model compartments and flows.

The four compartment $S I_{1} I_{2} A$ model adopted here is specified as follows, with migration flows of $M_{1}, M_{2}$, $M_{3}$ and $M_{4}$ to all of the four compartments. Let $S$ be the number of susceptible individuals in the population, $I_{1}$ represent the number of susceptible individuals who become exposed (infected but infectious), $I_{2}$ denote the number infected in the population, and $A$ denote the number of individuals having AIDS. The size of the individual population is therefore given by $N=S+I_{1}+I_{2}+A$. The disease occurs with equal probability across all age groups and, therefore, the incidence of natural death is constant across all compartments of the disease in individuals. In 2009, [9] formulated the spread of the AIDS epidemic with immigration of HIV infectives. There is a constant flow of immigration of susceptible individuals and individuals of the HIV class. Unlike the model from [9], in our model, the migrating individuals move into all of the four compartmental stages. For example, we include the novel feature of new born cases of infected individuals $b\left(I_{1}+I_{2}+A\right)$, for which we assume that $I_{1}, I_{2}$ and $A$ are sexual active, and $b \delta\left(I_{1}+I_{2}+A\right)$ are individuals who are infected and enter the exposed but 
infective stage. The remaining $(1-\delta) b\left(I_{1}+I_{2}+A\right)$ are susceptible individuals, where $(0<\delta<1)$ [10]. Infected individuals die at a rate $\mu$ as a result of the disease. $\beta_{1}, \beta_{2}$ and $\beta_{3}$ are the contact rates of susceptible with exposed, HIV and AIDS individuals, respectively, as indicated by the dotted lines shown in Figure 1.

With these assumptions regarding the dynamics of the individual populations and based on the Law of Mass Action are described by the following set of nonlinear differential equations:

$$
\begin{aligned}
& S^{\prime}=M_{1}+b S+(1-\delta) b\left(I_{1}+I_{2}+A\right)-\beta_{1} S I_{1}-\beta_{2} S I_{2}-\beta_{3} S I_{3}-\mu S \\
& I_{1}^{\prime}=M_{2}+b \delta\left(I_{1}+I_{2}+A\right)+\beta_{1} S I_{1}+\beta_{2} S I_{2}+\beta_{3} S I_{3}-(\alpha+\mu+\varepsilon) I_{1} \\
& I_{2}^{\prime}=M_{3}+\alpha I_{1}-(\rho+\mu+\gamma) I_{2} \\
& A^{\prime}=M_{4}+\rho I_{2}-(\mu+\sigma) A,
\end{aligned}
$$

where $M_{T}=M_{1}+M_{2}+M_{3}+M_{4}$, and $M_{i}(i=1,2,3,4)$ are the migrating individuals in the classes of susceptible, exposed (but infectious), HIV and AIDS, respectively. $\varepsilon, \gamma$ and $\alpha$ are the disease-induced death rates of the exposed-but-infectious, HIV-infected and AIDS-suffering individuals, respectively. The total population size can be determined by $N=S+I_{1}+I_{2}+A$ or from the differential equation $N^{\prime}=M_{T}+b N+\varepsilon I_{1}-\gamma I_{2}-\sigma A$. From the total population size, we have

$$
\begin{aligned}
N^{\prime} & =M_{T}+b N+\varepsilon I_{1}-\gamma I_{2}-\sigma A \\
& <M_{T}+b N+\varepsilon I_{1}-\gamma I_{2}-\sigma A
\end{aligned}
$$

By solving the second line of (2), we obtain:

$$
0 \leq N \leq \frac{M_{T}}{\mu-b}+N(0) e^{-(b+\mu) t},
$$

where $N(0)$ represents the initial values of respective variables. Then, $0 \leq N \leq \frac{M_{T}}{\mu-b}$ as $t \rightarrow \infty$. Hence, $\frac{M_{T}}{\mu-b}$ is an upper bound of $N$, provided that $N(0) \leq \frac{M_{T}}{\mu-b}$. If $0 \leq N(0)>\frac{M_{T}}{\mu-b}$ then $N(t)$ will decrease to this level. Therefore, all feasible solution of the system enters the region $\Psi=\left\{\left(S, I_{1}, I_{2}, A\right) \in \mathbb{R}_{+}^{4}: N \leq \frac{M_{T}}{\mu-b}\right\}$. See [9, 16] for more information.

\section{Equilibrium and stability analysis}

In this section, we analyse the existence and stability of the equilibrium points of the model system (1). It is important to determine whether the disease is epidemic or endemic; to determine this, the equilibrium point of the disease model where there is disease free equilibrium must be determined.

\subsection{Equilibrium of the model}

The model system (1) does not exhibit a disease free equilibrium due to the direct inflow of migrations at all four stages. However, one non-negative equilibrium point of the model (1) exists. This endemic equilibrium $E^{*}=\left(N^{*}, I_{1}^{*}, I_{2}^{*}, A^{*}\right)$ exists when HIV infection persists in the population, i.e. $I_{1} \neq 0$, where $N^{*}, I_{1}^{*}, I_{2}^{*}$ and $A^{*}$ are the positive solutions for the following algebraic equations:

$$
\begin{aligned}
M_{T}+b N+\varepsilon I_{1}-\gamma I_{2}-\sigma A & =0 \\
M_{2}+b \delta\left(I_{1}+I_{2}+A\right)+\beta_{1}\left(N-I_{1}-I_{2}-A\right) I_{1}+\beta_{2}\left(N-I_{1}-I_{2}-A\right) I_{2} & \\
+\beta_{3}\left(N-I_{1}-I_{2}-A\right) I_{3}-(\alpha+\mu+\varepsilon) I_{1} & =0 \\
M_{3}+\alpha I_{1}-(\rho+\mu+\gamma) I_{2} & =0 \\
M_{4}+\rho I_{2}-(\mu+\sigma) A & =0,
\end{aligned}
$$


which are all positive when $N<\frac{M_{T}}{\mu-b}$. By solving (4) simultaneously, we obtain:

$$
\begin{aligned}
& I_{1}=\frac{\rho+\mu+\gamma}{\alpha}\left[\frac{\alpha(\mu+\sigma)\left(M_{T}+b N-\mu N\right)+\varepsilon(\mu+\sigma) M_{3}-\sigma \alpha M_{4}}{\varepsilon(\mu+\sigma)(\rho+\mu+\gamma)+\gamma \alpha(\mu+\sigma)+\alpha \sigma \rho}\right]-\frac{M_{3}}{\alpha} \\
& I_{2}=\frac{\alpha(\mu+\sigma)\left(M_{T}+b N-\mu N\right)+\varepsilon(\mu+\sigma) M_{3}-\sigma \alpha M_{4}}{\varepsilon(\mu+\sigma)(\rho+\mu+\gamma)+\gamma \alpha(\mu+\sigma)+\alpha \sigma \rho} \\
& A=\frac{\rho+\mu+\gamma}{\mu+\sigma}\left[\frac{\alpha(\mu+\sigma)\left(M_{T}+b N-\mu N\right)+\varepsilon(\mu+\sigma) M_{3}-\sigma \alpha M_{4}}{\varepsilon(\mu+\sigma)(\rho+\mu+\gamma)+\gamma \alpha(\mu+\sigma)+\alpha \sigma \rho}\right]
\end{aligned}
$$

From the second line of (4), the existence of $E^{*}$ is shown below

$$
\begin{array}{r}
F(N)=M_{2}+b \delta\left(I_{1}+I_{2}+A\right)+\beta_{1}\left(N-I_{1}-I_{2}-A\right) I_{1}+\beta_{2}\left(N-I_{1}-I_{2}-A\right) I_{2} \\
+\beta_{3}\left(N-I_{1}-I_{2}-A\right) I_{3}-(\alpha+\mu+\varepsilon) I_{1}
\end{array}
$$

From (5), $N$ has a positive root interval between 0 and $\frac{M_{T}}{\mu-b}$. When $N=0$, (5) becomes:

$$
\begin{array}{r}
F(0)=M_{2}+b \delta\left(I_{1}+I_{2}+A\right)-\beta_{1}\left(I_{1}+I_{2}+A\right) I_{1}-\beta_{2}\left(I_{1}+I_{2}+A\right) I_{2} \\
-\beta_{3}\left(I_{1}+I_{2}+A\right) A-(\alpha+\mu+\varepsilon) I_{1},
\end{array}
$$

and when $N=\frac{M_{T}}{\mu-b}$ we have

$$
\begin{array}{r}
F\left(\frac{M_{T}}{\mu-b}\right)=M_{2}+b \delta\left(I_{1}+I_{2}+A\right)+\beta_{1}\left(\frac{M_{T}}{\mu-b}-\right. \\
\left.I_{1}-I_{2}-A\right) I_{1}+\beta_{2}\left(\frac{M_{T}}{\mu-b}-I_{1}-I_{2}-A\right) I_{2} \\
+\beta_{3}\left(\frac{M_{T}}{\mu-b}-I_{1}-I_{2}-A\right) A-(\alpha+\mu+\varepsilon) I_{1} .
\end{array}
$$

Thus,

$$
F^{\prime}(N)=\beta_{1} I_{1}+\beta_{2} I_{2}+\beta_{3} A
$$

\subsection{Local stability of the equilibrium point}

The stability of the endemic equilibrium is determined using the eigenvalues of the characteristic equation of the corresponding Jacobian matrix, $\mathbb{J}\left(N^{*}, I_{1}^{*}, I_{2}^{*}, A^{*}\right)=\mathbb{J}\left(E^{*}\right)$, which is given by:

$$
\mathbb{J}\left(E^{*}\right)=\left(\begin{array}{cccc}
n_{1} & -\varepsilon & -\gamma & -\sigma \\
b \delta & n_{2} & \beta_{2} S & \beta_{3} S \\
0 & \alpha & -n_{3} & 0 \\
0 & 0 & \rho & -n_{4}
\end{array}\right),
$$

where $n_{1}=b-\mu, n_{2}=\beta_{1} S, n_{3}=-(\rho+\mu+\sigma)$ and $n_{4}=-(\mu+\sigma)$. The characteristic equation corresponding to $\mathbb{J}\left(E^{*}\right)$, is given by

$$
f(\lambda)=\lambda^{4}+a_{1} \lambda^{3}+a_{2} \lambda^{2}+a_{3} \lambda^{1}+a_{4}=0 .
$$

$$
\begin{aligned}
& a_{1}=n_{3}+n_{4}-n_{1}-n_{2}, \\
& a_{2}=\varepsilon b \delta-\alpha \beta_{2} S+n_{1} n_{2}-n_{1} n_{3}-n_{1} n_{4}-n_{2} n_{4}+n_{3} n_{4}, \\
& a_{3}=\alpha \beta_{2} n_{1} S-\alpha \beta_{2} n_{4} S+\alpha b \delta \gamma-\alpha \beta_{3} \rho S+\varepsilon b \delta n_{4}+n_{1} n_{2} n_{3}+n_{1} n_{2} n_{4}-n_{1} n_{3} n_{4}-n_{2} n_{3} n_{4}, \\
& a_{4}=\alpha b \delta n_{4} \gamma+\alpha b \delta \rho \gamma+\alpha \beta_{3} n_{1} \rho S+\alpha \beta_{2} n_{1} \rho S+\alpha \beta_{2} n_{1} n_{4} \rho S+\varepsilon b \delta n_{4}+n_{1} n_{2} n_{3} n_{4} .
\end{aligned}
$$


Because (10) is a polynomial of degree four [27, 16], from the Routh-Hurwitz Theorem, the endemic equilibrium will be stable if $a_{1}>0, a_{2}>0, a_{3}>0$, and $a_{1} a_{2} a_{3}>a_{3}^{2}+a_{4}$. From this condition of stability, we have

$$
\begin{aligned}
& a_{1}:\left(n_{3}+n_{4}>n_{1}+n_{2}\right), \\
& a_{2}:\left(\varepsilon b \delta-\alpha \beta_{2} S+n_{1} n_{2}-n_{1} n_{3}-n_{1} n_{4}-n_{2} n_{4}+n_{3} n_{4}\right), \\
& a_{3}:\left(\alpha \beta_{2} n_{1} S+\alpha b \delta \gamma+\varepsilon b \delta n_{4}+n_{1} n_{2} n_{3}+n_{1} n_{2} n_{4}>\alpha \beta_{2} n_{4} S+\alpha \beta_{3} \rho S+n_{1} n_{3} n_{4}+n_{2} n_{3} n_{4}\right), \\
& a_{4}:\left(\alpha b \delta n_{4} \gamma+\alpha b \delta \rho \gamma+\alpha \beta_{3} n_{1} \rho S+\alpha \beta_{2} n_{1} \rho S+\alpha \beta_{2} n_{1} n_{4} \rho S+\varepsilon b \delta n_{4}+n_{1} n_{2} n_{3} n_{4}>0\right),
\end{aligned}
$$

which indicates that the endemic equilibrium is stable.

To determine whether the disease continues to spread, we must find the stability of the disease free equilibrium point. The reproduction number $R_{0}$ is the expected number of secondary cases produced by a single infection in a completely susceptible population [15]. We have already observed that the disease cannot persist if $R_{0}<1$. The condition $R_{0}<1$ corresponds to the local as well as the global stability of the disease free equilibrium. Thus, the disease will not continue to spread if $R_{0}<1$. To determine whether the disease will continue to spread, we must study the dynamics for $R_{0}>1$. If the disease persists in the system $\left(R_{0}>1\right)$, then either the system will be stable around the interior equilibrium or there may exist a periodic attractor. Using the notations by van den Driessche and Watmough [18], $F_{i}(x)$ is the rate of appearance of new infections in compartment $i$, and $V_{i}(x)$ represents the rate of infections from one compartment to another; the corresponding Jacobian matrices for $F_{i}$ and $V_{i}$ are given by

$$
\begin{gathered}
F=\left(\begin{array}{ccc}
b \delta+\beta_{1} & b \delta+\beta_{2} & b \delta+\beta_{3} \\
0 & 0 & 0 \\
0 & 0 & 0
\end{array}\right), \\
V=\left(\begin{array}{ccc}
\alpha+\mu+\varepsilon & 0 & 0 \\
0 & \rho+\mu+\gamma & 0 \\
0 & 0 & \mu+\sigma
\end{array}\right),
\end{gathered}
$$

The next generation matrix $F V^{-1}$, where $F$ is non-negative and $V$ is a non-singular matrix is given by

$$
F V^{-1}=\left(\begin{array}{ccc}
\frac{b \delta+\beta_{1}}{\alpha+\mu+\varepsilon} & \frac{b \delta+\beta_{2}}{\rho+\mu+\gamma} & \frac{b \delta+\beta_{3}}{\mu+\sigma} \\
0 & 0 & 0 \\
0 & 0 & 0
\end{array}\right)
$$

The basic reproduction number $R_{0}$ that describes endemic persistence of the disease is the spectral radius of $F V^{-1}$. Hence, at disease free equilibrium,

$$
R_{0}=\frac{b \delta+\beta_{1}}{\alpha+\mu+\varepsilon} .
$$

\subsection{Without Immigration}

In a situation where there is no migration, we have $M_{T}=0$ and (4) correspond to (15).

$$
\begin{aligned}
b N+\varepsilon I_{1}-\gamma I_{2}-\sigma A & =0 \\
b \delta\left(I_{1}+I_{2}+A\right)+\beta_{1}\left(N-I_{1}-I_{2}-A\right) I_{1}+\beta_{2}\left(N-I_{1}-I_{2}-A\right) I_{2} & \\
+\beta_{3}\left(N-I_{1}-I_{2}-A\right) I_{3}-(\alpha+\mu+\varepsilon) I_{1} & =0 \\
\alpha I_{1}-(\rho+\mu+\gamma) I_{2} & =0 \\
\rho I_{2}-(\mu+\sigma) A & =0,
\end{aligned}
$$


From the third and fourth line of (15), we have

$$
\begin{gathered}
I_{1}=\frac{(\rho+\mu+\gamma) I_{2}}{\alpha}, \\
A=\frac{\rho I_{2}}{\mu+\gamma} .
\end{gathered}
$$

For disease free $E^{*}(N, 0,0,0)$, from second line of (15), we obtain

$$
S^{0}=\frac{b N(1-\delta)}{\mu-b \delta} .
$$

We define the reproduction number $[9,15,18,21,16,17]$ from $(18)$ as

$$
R_{01}=\frac{\mu-b \delta}{b N(1-\delta)}
$$

The total population is $S^{0}+I_{1}^{0}+I_{2}^{0}+A^{0}=N$ with all positive values, where

$$
\begin{aligned}
I_{1}^{0} & =\frac{(\rho+\mu+\gamma)(\mu+\sigma)\left(R_{01} N-1\right)}{R_{01}[(\mu+\sigma)(\rho+\mu+\gamma)+\alpha(\mu+\sigma+\rho)]}, \\
I_{2}^{0} & =\frac{\alpha(\mu+\sigma)\left(R_{01} N-1\right)}{R_{01}[(\mu+\sigma)(\rho+\mu+\gamma)+\alpha(\mu+\sigma+\rho)]}, \\
A^{0} & =\frac{\rho \alpha(\mu+\sigma)\left(R_{01} N-1\right)}{R_{01}[(\mu+\sigma)(\rho+\mu+\gamma)+\alpha(\mu+\sigma+\rho)]}
\end{aligned}
$$

\section{Experimental and results analysis}

We will use prevalence rate of HIV/AIDS by dividing the total number of cases of a disease existing in a population by the total population. The prevalence rate of adults among the population with HIV and AIDS is between 15 to 49 percent, according to UNAIDS/WHO [22]. The $S I_{1} I_{2} A$ model considers only adults in the population compartments. The UN population reported in 1990, there were 15.190 million individuals in Ghana and the adult population, i.e., ages 15 to 64 years [23], was 53\%; this corresponds to 8,050,700 individuals for the starting susceptible and HIV, class for that year. During that time, the adult prevalence rate was approximately $0.2 \%$ [23], which corresponds to $16,101.4$ individuals for the starting infected $I_{2}(0)$ compartment. Thus, there are 8,034,599 individuals in the susceptible $S(0)$ class (similar method was by [24].

Based on the above information, the following sets of initial conditions are established, as presented in the Table 1 and Table 2. A spectral analysis is used to ascertain the differences observed in the various stages of the spread of the disease. This time series procedure is suitable to determine the peak of the disease when the distributions are frequency dependent. However, given that analysed is distributed, the spectral analysis is the time series of choice. Appropriately, a plot of the variance versus frequency is used to determine the speed at which the disease spreads; this analysis is also called the periodogram analysis. By using $1 /$ frequency, the rate at which the disease spreads is estimated. We solve the set of differential equations using the odesolve package in R-project [30].

The values in Table 2 were based on an assumption. We assumed that individuals in the exposed (but infectious) stage will be more sexually active as compare to those in the HIV and AIDS stages $\beta_{1}>\beta_{2}>\beta_{3}$. The assumption is based on the following reasons: individuals at the exposed (but infectious) stage are more sexual active because when people become infected with HIV, the people do not develop symptoms of AIDS for 10 to 12 years. We assumed that $\varepsilon$ out $0.5 \%$ of people who are exposed (but infectious) will die due to the disease. 
Table 1. Model parameters definitions

\begin{tabular}{|l|l|l|}
\hline Parameter & Definition & Values \\
\hline$M_{n}, n=1, . ., 4$ & Migration rate & $0.00223[13]$ \\
\hline$\mu$ & Natural death rate & $0.00783[25]$ \\
\hline$b$ & Natural birth rate & $0.0321[5,26]$ \\
\hline$\alpha$ & Rate of movement from $I_{1} \& I_{2}$ & $0.15[26]$ \\
\hline$\rho$ & Rate of movement from $I_{2} \& A$ & $0.018[26]$ \\
\hline$\gamma \& \sigma$ & Disease-induced death rates of $I_{2} \& A$ & $0.0007563[29]$ \\
\hline
\end{tabular}

Table 2. Model parameters definitions

\begin{tabular}{|l|l|l|}
\hline Parameter & Definition & Assumed values \\
\hline$\beta_{1}$ & Contact rate between $S$ and $I_{1}$ & 0.9 \\
\hline$\beta_{2}$ & Contact rate between $I_{1}$ and $I_{2}$ & 0.6 \\
\hline$\beta_{3}$ & Contact rate between $I_{2}$ and $A$ & 0.1 \\
\hline$\delta$ & Fraction of $S$ not infected & 0.006 \\
\hline$\varepsilon$ & Disease-induced death rates of $I_{1}$ & 0.005 \\
\hline
\end{tabular}

The results of a numerical simulation based on literature sources and assumptions are shown in Figures 2 - 11 . Figure 2 shows the distribution of susceptible population with time during the flow of external migration, while Figure 3 shows the results when there was no flow of migration into the susceptible stage.

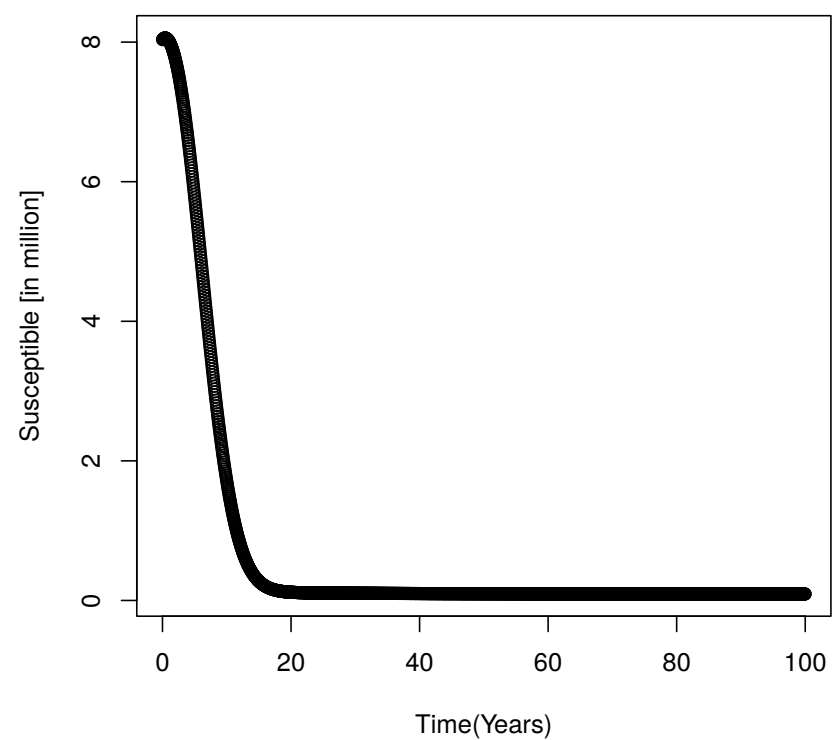

Figure 2. Susceptible population with migration.

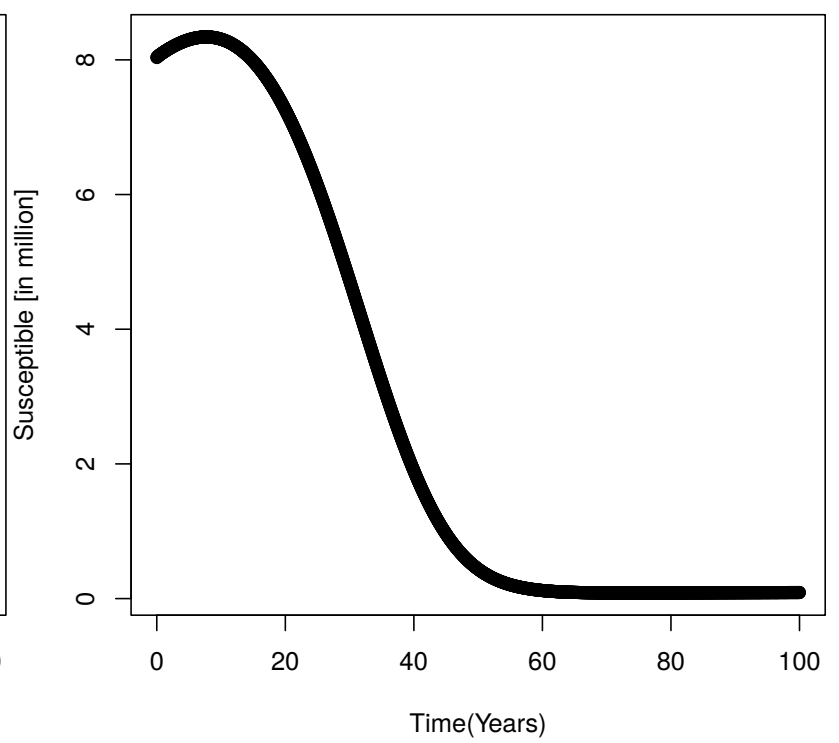

Figure 3. Susceptible population without migration.

The constant flow of migration into the susceptible population, the new born cases of infected individuals entered to the exposed (but infectious) population. The susceptible population first increases with an increase in time and reaches an equilibrium peak. There is a sharp decrease in the population of the susceptible population. For example, in Figure 2, with migration, there are 117548, 990975 and 931088 people at 20, 60 and 100 years, respectively, while without migration, at the same periods of 20,60 and 100 years are 7.256753 million, 119714 and 90173 people, respectively, in Figure 3. Thus, the number of susceptible individuals decreases significantly as the prevalence continues to increase. The disease still continues to remain endemic in 
the population at a high prevalence because the infected individuals continue to infect more susceptible individuals.

Figure 4 depicts the distribution of the exposed (but infectious) population with time including the flow of external migration, while Figure 5 shows the distribution with no flow of migration into the exposed (but infectious) stage.

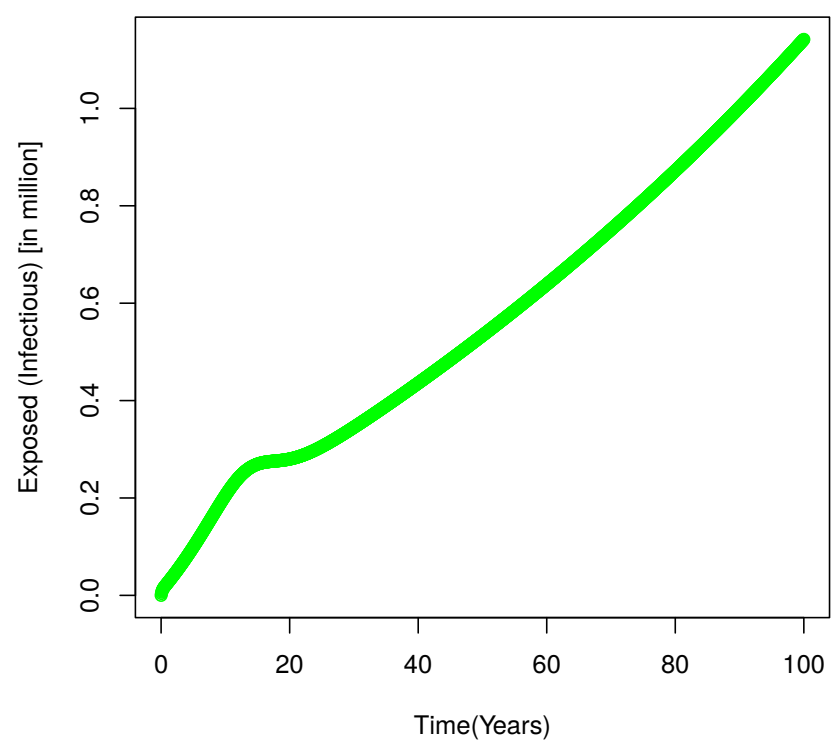

Figure 4. Exposed population with migration flow.

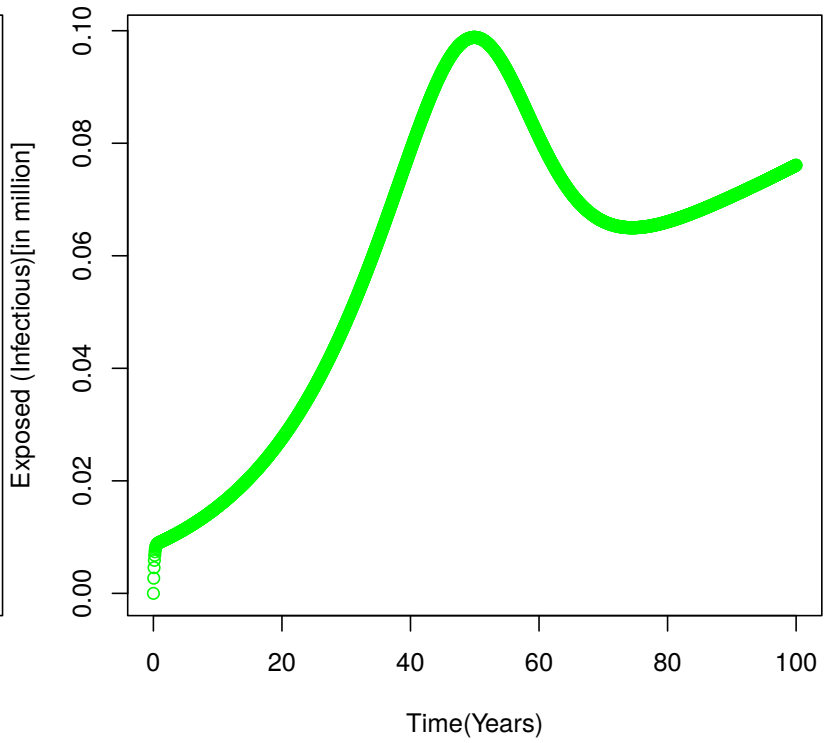

Figure 5. Exposed population without migration flow.

In Figure 4, a constant increase in the exposed population was calculated between year 20 and year 100, corresponding to 280006 and 1.141756 million people exposed, respectively. In Figure 5, there is increase in the period from 20 years to 50 years up to the peak of those who were exposed but infectious at 98816 , followed by a decrease to 66045 people at 80 years. Thus, the infected new-born children enter directly into increase of the exposed (but infectious) population. However, the new born children can be controlled by preventive measures, such as promoting the use of condoms to minimise the spread of the disease.

Figure 6 depicts the distribution of the population with HIV disease with time with the flow of external migration, while Figure 7 shows the case with no flow of migration into the population in the HIV stage.

With the constant flow of migration, the HIV population constantly increased throughout period of the simulation. For example, in Figure 6, with migration flow, at 20 years and 100 years, the HIV population is 818524 and 4.884736 million people, respectively, whereas in Figure 7, for the case with no migration flow, at 20 years and 100 years, the HIV population is 49209 thousand and 364229 thousand people, respectively. To minimise the spread of HIV, the movement rate of $\alpha$ must be increased to reduce the number of infected individuals who will move into the AIDS population. Thus, the movement rate of is the major contributing factor of the spread of the disease.

Figure 8 depicts the distribution of the population with the AIDS disease with time including the flow of external migration, while Figure 9 considers the case with no flow of migration into the AIDS stage.

In Figure 8, a constant increase in the population with AIDS is simulated between 20 years and 100 years, corresponding to 552228 and 4.669207 million AIDS patients, respectively. In Figure 9, there is slight increase from 20 years to 40 years, followed by a sharp increase to 100 years, with the number of AIDS patients of 9879 , 39109 and 285813 people at years 20,40, and 100, respectively. To minimise the spread of AIDS, the movement rate of $\rho$ and $\sigma$ must be increased to reduce the number of infected individuals who will transfer into the AIDS 


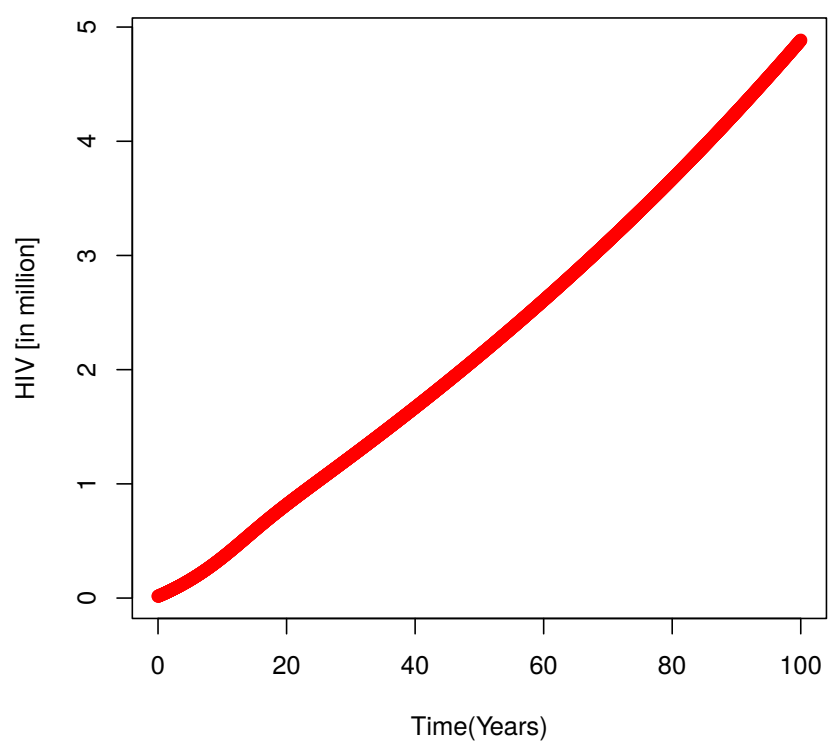

Figure 6. HIV population with migration flow.

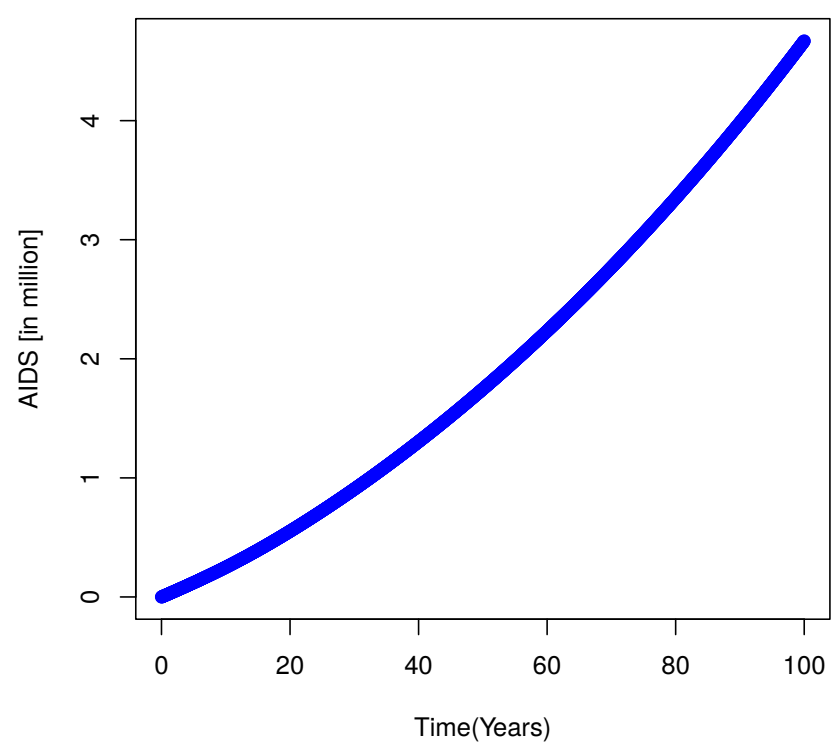

Figure 8. AIDS population with migration flow.

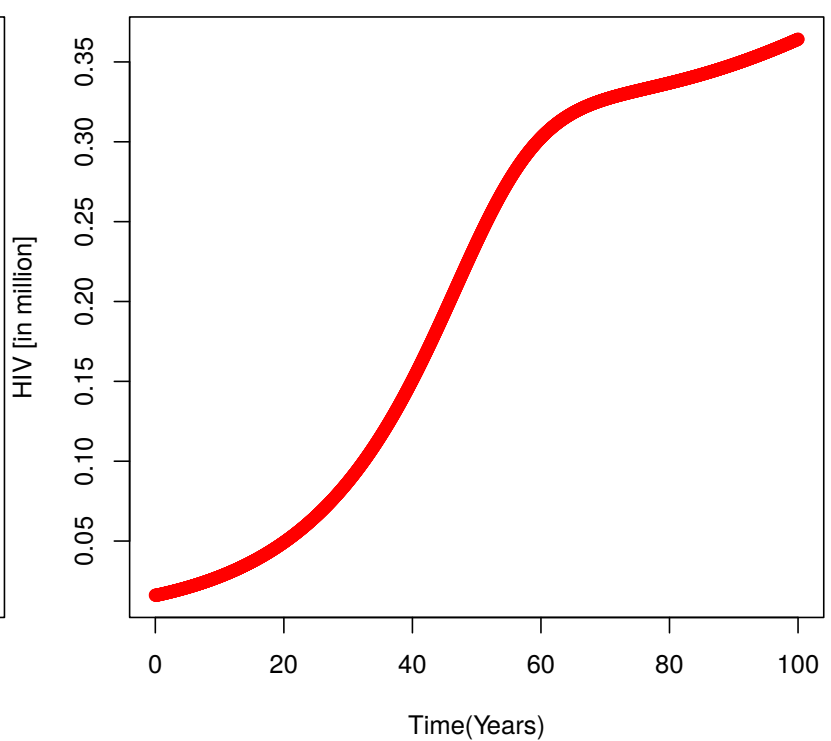

Figure 7. HIV population without migration flow.

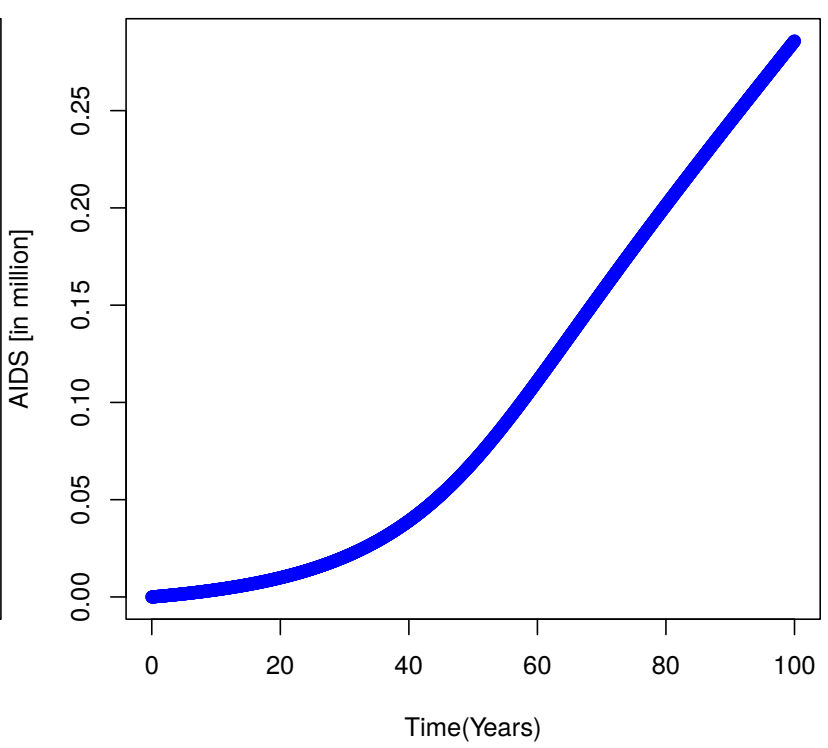

Figure 9. AIDS population without migration flow.

population. Thus, the movement rate of $\alpha$ and $\sigma$ are the major contributing factor of the increase of the AIDS population.

Figure 10 depicts the significance of each frequency, as measured by the spectral amplitude. The periodogram analysis of the disease shows how the rate at which the disease spreads depends on the persistent flow of migration. The contour plot (Figure 11) shows that there is widespread HIV affecting many persons at 2 years intervals and that the spread from person to person is periodic 

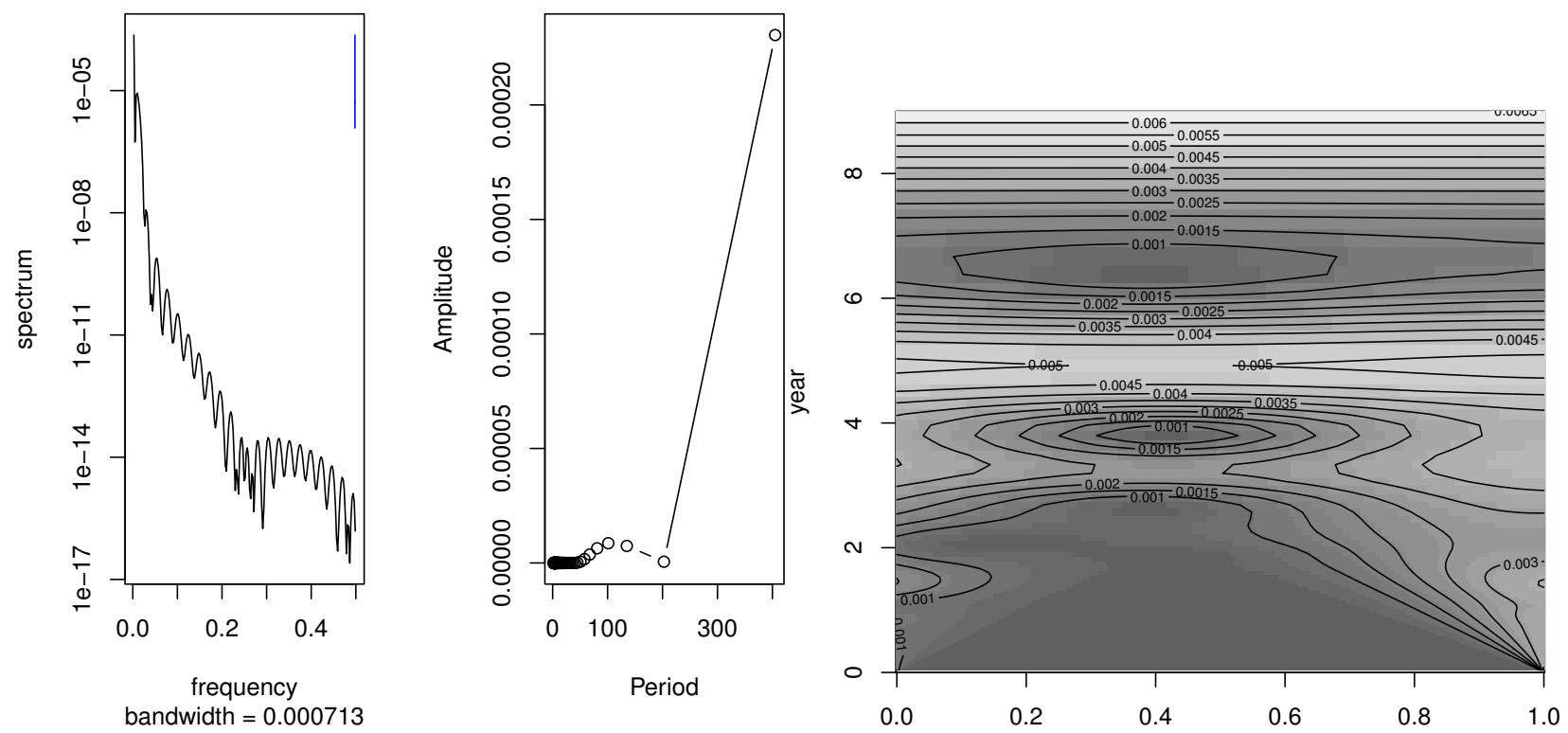

Figure 10. Periodogram of the HIV population with migration Figure 11. Interepidemic plot of the HIV population with flow. migration flow.

\section{Conclusion}

In this paper, we modelled the impact of the persistent flow of migration on the population with HIV. We assumed that there were sexual interactions between the susceptible and HIV population and that the infected new-born babies were moved directly to the acute stage to increase the growth of the exposed (but infectious) population. It is also assumed that people in the exposed (but infectious), HIV and AIDS classes in decreasing order are capable of giving birth. If $R_{0}>1$, then an epidemic of AIDS occurs, and if $R_{0}<1$, then the disease becomes endemic. From the analysis of the model, the reproduction number is used to determine whether the disease is epidemic or endemic. We found that $R_{01}=2.9795 \times 10^{-5}$ for the case without the flow of migration, which indicates the disease is endemic, while for the case with the persistent flow of migration into the country, the HIV disease has become epidemic, with $R_{0}=5.53287$. Which is not a good indicator of the public health sector. This is because in case of any infectious disease there is the need to either eradicate or maintain a stable stage of the disease. Figures 2-5 and 7 show the populations of the respective individuals at their equilibrium levels. The rate of speed at which the disease was spread with the flow of constant migration into the four compartmental stages was found to be 1403 seconds (Figure 10). The simulation results indicated that by controlling the migration flow into the population stages (Figures 5, 7 and 9), the spread of the disease can be minimised significantly. To reduce the spread of the disease, the flow of migration can be restricted. Periodically, every 2 years, the flow of migration can be investigated because the spread of disease is at its peak stage. Further study is necessary to obtain realistic parameter values. Having those challenges in mind, these results require further investigation before being used as a guide for early treatment of infected individuals, but the results do suggest an emphasis on prevention policies that reduce migration policy.

\section{REFERENCES}

1. UNAIDS report on the global AIDS epidemic, Global Report, 2013.

2. S. G. Deeks, S. R.Lewin, and D. V. Havlir, The end of AIDS: HIV infection as a chronic disease, The Lancet, 382(9903), pp.1525$1533,2013$.

3. K. S. Khan, D.Wojdyla, L. Say, A. M.Glmezoglu, and P. F. Van Look. WHO analysis of causes of maternal death: a systematic review, The lancet, 367(9516), pp.1066-1074, 2006. 
4. C. D. Mathers and , D. Loncar, Projections of global mortality and burden of disease from 2002 to 2030. PLoS medicine, 3(11), e442, 2006

5. Brummer, D. Labour Migration and HIV/AIDS in Southern Africa, International Organisation for Migration Regional Office for Southern Africa. pp. 1-26. 2002

6. M. Coffee, M.N. Lurie, and G.P. Garnett, Modelling the impact of migration on the HIV epidemic in South Africa, AIDS, 21(3): pp. 343-350, 2007.

7. K. N. Deering, P. Vickerman, S. Moses, B. M. Ramesh, J. F. Blanchard and M. C. Boily, The impact of out-migrants and outmigration on the HIV/AIDS epidemic: a case study from south-west India. AIDS, 22: pp. 165-181, 2008.

8. E. A. Parrado, C. A. Flippen, and C. McQuiston, Use of commercial sex workers among Hispanic migrants in North Carolina: implications for the spread of HIV, Perspectives on sexual and reproductive health, 36(4), pp.150-156, 2004.

9. R. Naresh, A. Tripathi, and S. Omar, Modelling the spread of AIDS epidemic with vertical transmission, Applied Mathematics and Computation, 178(2): pp. 262-272, 2006.

10. O. O. Apenteng and Ismail, N. A. Ismail, A Markov Chain Monte Carlo Approach to Estimate AIDS after HIV Infection, PloS one, 10(7), e0131950, 2015.

11. L. Corno and D. De Walque, Mines, migration and HIV/AIDS in Southern Africa, The World Bank, 2012.

12. M. T Mbizvo and M. T Bassett, Reproductive health and AIDS prevention in sub-Saharan Africa: the case for increased male participation, Health Policy and Planning, 11(1), pp.84-92, 1996.

13. P. Quartey, Migration in Ghana: A Country profile 2009, 2010.

14. UNHCR, Global Trends of asylum of refugees 2012: Table 18. Major mass inflows, 2012.

15. J. H. Jones, Notes On RO, Department of Anthropological Sciences: Califonia. pp. 17, 2007.

16. O. O. Apenteng and , N. A.Ismail, Modelling the spread of HIV and AIDS epidemic trends in male and female populations, World Journal of Modelling and Simulation, 13(3), pp.183-192, 2017.

17. S. Rosa and D. F Torres, Parameter Estimation, Sensitivity Analysis and Optimal Control of a Periodic Epidemic, Model with Application to HRSV in Florida. pp.140-149, 2018.

18. P. van den Driessche and J. Watmough, Reproduction numbers and sub-threshold endemic equilibria for compartmental models of disease transmission, Mathematical Biosciences, 180(1-2): pp. 29-48, 2005.

19. R. Naresh, A. Tripathi, and D. Sharma, A nonlinear HIV/AIDS model with contact tracing, Applied Mathematics and Computation, 217(23): pp. 9575-9591, 2011.

20. J. M. Gran, L.Wasmuth, E. J.Amundsen, B. H. Lindqvist, and O. O.Aalen, Growth rates in epidemic models: Application to a model for HIV/AIDS progression, Statistics in Medicine, 27(23): pp. 4817-4834, 2008.

21. T. Zhang, M. Jia, H. Luo, Y. Zhou, and N. Wang, Study on a HIV/AIDS model with application to Yunnan province, China Applied Mathematical Modelling, 35(9): pp. 4379-4392, 2011.

22. UNAIDS/WHO: Adult (15-49) HIV prevalence percent by country, 1990-2007, 2008 Report on the global AIDS epidemic, 2008.

23. A. Adlakha, International Brief. Population trends: Ghana, Department of Commerce United State, 2006.

24. F. Nyabadza, Z. Mukandavire, and S.D. Hove-Musekwa, Modelling the HIV/AIDS epidemic trends in South Africa: Insights from a simple mathematical model, Nonlinear Analysis: Real World Applications, 12(4): pp. 2091-2104, 2011.

25. Ghana - Death rate, crude, (per 1;000 People) in Ghana, http://www.tradingeconomics.com/ghana/death-rate-crude-per-1-000people-wb-data.html, 2014.

26. UNICEF. State of the Worlds Children, Statistical Tables, http://www.unicef.org/sowc2011/pdfs/SOWC-2011-Statistical-tables12082010.pdf, 2011

27. A. Galindro, and D. F.Torres, A simple mathematical model for unemployment: a case study in Portugal with optimal control. arXiv preprint arXiv:1801.00058, 2017.

28. R. Naresh, A. Tripathi, and D. Sharma, Modelling and analysis of the spread of AIDS epidemic with immigration of HIV infectives, Mathematical and Computer Modelling, 49: pp. 880-892, 2009.

29. Comprehensive, Up-to-date Information on HIV/AIDS Treatment, Prevention, and Policy University of California San Francisco, http://hivinsite.ucsf.edu/global?page=cr09-gh-00, 2013

30. Team, R. C. R: A language and environment for statistical computing, 2013 